Pacific Journal of Mathematics

A THEOREM ON NOETHERIAN HEREDITARY RINGS 


\title{
A THEOREM ON NOETHERIAN HEREDITARY RINGS
}

\author{
Victor P. CAMillo AND J. Cozzens
}

\begin{abstract}
It is shown (Theorem 2) that a semi-prime, left noetherian, left hereditary, two-sided Goldie ring is right noetherian if and only if the right module $(Q / R) \oplus R$ contains a copy of every simple right $R$-module, where $Q$ is the classical quotient ring of $R$. Theorem 5 gives several necessary and sufficient conditions for a semi-prime principal left ideal ring which is right Goldie to be a principal right ideal ring. Among these is that $R / A$ must be artinian for every essential left ideal $A$.

It is known that a two-sided noetherian semi-prime ring is principal on the left if and only if it is principal on the right. On the other hand, if one drops the ascending chain condition on the right side of $R$, examples are known of principal left ideal domains (p.1.i. domains) which are not right principal. But, if we require that they be right Ore as well, things may be better.
\end{abstract}

We have not been able to produce an example of a principal left ideal domain which is not right principal, but if such an example exists it must have more than one simple right $R$-module. This problem is also mentioned in Cohn's new book, Free Rings and Their Relations.

I. A theorem on hereditary noetherian rings. All rings will be understood to be associative with identity element. Mod- $R$ (resp., $R$-mod) will denote the category of all right $R$-modules (left $R$-modules). ${ }_{R} A\left(A_{R}\right)$ will denote the fact that $A$ belongs to $R$-mod $(\bmod -R)$. A left (right) Ore domain is an integral domain (i.e., a ring without divisors of zero) which possesses a classical left (right) quotient ring which is necessarily a division ring (see [4]). A ring $R$ is left hereditary $\left(\chi_{0}\right.$ hereditary, semihereditary) if each left ideal (countably generated left ideal, finitely generated left ideal) is projective in $R$-mod. Similar definitions hold on the right. Some of the ideas occurring in the proof of Theorem 2 stem from the paper by Webber [9].

LEMma 1. Let $R$ be right semi-hereditary and right Goldie with two-sided classical quotient ring $Q$. Let $S$ be a simple right $R$-module. Then the following are equivalent:

1. $S$ is finitely presented.

2. $S$ may be embedded in the module $(Q / R) \oplus R$. In fact, under these conditions, $S$ either embeds in $Q / R$, or is a summand of $R$. 
Proof. If $S$ is finitely presented, there is an exact sequence:

$$
0 \longrightarrow K \longrightarrow P \longrightarrow S \longrightarrow 0 \text {, }
$$

with $P$ projective and $K$ finitely generated. We also have

$$
0 \longrightarrow M \longrightarrow R \longrightarrow S \longrightarrow 0 \text {, }
$$

for some maximal right ideal $M$. Then, by Shanuel's lemma [6, p. 167], $K \oplus R \approx P \oplus M$, so $M$ is finitely generated. In particular, $M$ is projective. We distinguish two cases:

Case I. $M$ is not large in $R$. Then there is an $x \in R, x \neq 0$ and $x R \cap M=0$. So $R=x R \oplus M$, with $x R \approx S$, and we are done.

Case $I I . M$ is large. Then, $M$ contains a regular element $c$. Also we may chose a dual basis $\left\{x_{i}, f_{i}\right\}$ for $M$. Now, $Q_{R}$ is injective [8] so that each $f_{i}$ is just left multiplication by some $q_{i} \in Q$. In particular,

or,

$$
\sum x_{i} q_{i} c=c,
$$

$$
\sum x_{i} q_{i}=1 \text {. }
$$

Since $M \neq R$ some $q_{i} \notin R$, but $q_{i} M \subset R$, so the map given by $1+$ $M \mapsto q_{i}+R$ is the desired embedding of $R / M$ into $Q / R$.

2 implies 1: Since $S$ is simple, the hypothesis implies that $S$ is embeddable in either $R$ or $Q / R$. If $S$ is a submodule of $R$, then the semi-primeness of $R$ forces $S$ to be generated by an idempotent. If $S$ is embeddable in $Q / R$, let $q M \subset R$ with $q \notin R$ and $S \approx R / M$. Then $q=b^{-1} a$, since the quotient ring is two-sided. But then $q R+R \subset$ $b^{-1} R \approx R$, and is thus projective. The exact sequence:

$$
0 \longrightarrow R \longrightarrow q R+R \longrightarrow S \longrightarrow 0
$$

gives the desired presentation.

THEOREM 2. Let $R$ be a left noetherian, left hereditary, semi-prime ring which is right (and hence two-sided) Goldie. Then the following are equivalent.

1. $R$ is right noetherian (and hence hereditary).

2. The module $Q / R \oplus R$ contains a copy of every simple right $R$-module.

Proof. 1 implies 2: Every simple module is finitely presented, and 2 follows from Lemma 1.

2 implies 1: Since $R$ is left hereditary and finite dimensional on the right, $R$ is right semi-hereditary by [3]. If $R$ is not right noetherian, 
let $A$ be a maximal nonfinitely generated right ideal of $R$. Clearly $A$ is an essential right ideal of $R$, and hence contains a regular element $c$ ([4], Lemma 2.12). We first show that $R / A$ is an artinian right $R$ module. Let

$$
A_{1} \supset A_{2} \cdots \supset A \supset c R
$$

be a descending chain. These are all finitely generated, hence projective.

We now use the fact that the functor $\operatorname{Hom}_{R}(-R)$ defines a category anti-equivalence between finitely generated left and right projectives to obtain a sequence

$$
0 \longrightarrow \operatorname{Hom}_{R}\left(A_{1}, R\right) \longrightarrow \operatorname{Hom}_{R}\left(A_{2}, R\right) \cdots
$$

where none of the maps are isomorphisms unless the chain stops. Now, we embed this sequence as a chain in $R$ by $f \mapsto f(c)$. This is a left $R$-module map, and is an embedding because $c R$ is large and the right singular submodule of $R$ is zero [i.e., if $f(c)=0, x \in$ Domain $f$, and $I=\{r \mid x r \in c R\}$, then $I$ is large, and $f(x) I=0]$.

This yields a strict ascending chain of left ideals which must stop, so that the original chain must also have stopped.

Now since $R / A$ is artinian it contains a simple submodule, say $A^{\prime} / A$. Now $A^{\prime}$ is projective and we have an exact sequence:

$$
0 \longrightarrow A \longrightarrow A^{\prime} \longrightarrow A^{\prime} / A \longrightarrow 0
$$

and by Lemma 1 an exact sequence

$$
0 \longrightarrow K \longrightarrow P \longrightarrow A^{\prime} / A \longrightarrow 0
$$

with $K$ finitely generated and $P$ projective. As in Lemma $1, A$ is finitely generated by Shanuel's lemma.

II. We now apply the results of the previous section to obtain information about the right side of a two-sided Goldie ring which is semi-prime and left principal.

LEMmA 3. Let $R$ be left (or right) Goldie and let $x$ be a non-zero divisor in $R$, then there is an anti-isomorphism of partially ordered sets between principal left ideals containing $x$ and principal right ideals containing $x$.

Proof. Let $u R$ be a right ideal containing $x$. Then, since $x=$ $u r$ for some $r, u$ is left regular. Since $R$ is an order in a semi-simple ring, a left regular element must be right regular, because such an element will have a two-sided inverse in the quotient ring. Thus, $u$ is right regular. 
The isomorphism $H$ is defined as follows: If $u R$ is a right ideal containing $x$, define $H(u R)=R v$, where $x=u v$. There are two problems of well-definedness:

I. If $u R=w R$, then $u=w \alpha$ where $\alpha$ is a unit, since $u$ and $w$ are regular. Then, $x=u v=w \alpha v$ and $R v=R \alpha v$, so $H$ is independent of the choice of the generator for $u R$.

II. If $x=u v=u v_{1}$, then $v=v_{1}$, since $u$ is right regular.

$H$ is trivially onto, and is one-to-one, since if $H(u R)=R v$ and $H\left(u^{\prime} R\right)=R v$, we have $x=u r_{1} v$ and $x=u^{\prime} r_{2} v$ where $R r_{1} v=R r_{2} v=R v$. In particular, $r_{1}$ and $r_{2}$ are units, and $u r_{1}=u^{\prime} r_{2}$, so $u R=u^{\prime} R$. One also checks easily that $H$ is order reversing.

The following lemma is surely in the literature, but we have been unable to locate it.

LEMma 4. Let $R$ be a semi-prime principal left ideal ring, then $R$ is left hereditary.

Proof. If $R x$ is a principal left ideal, then there is a left ideal $R h$ such that $R x \oplus R h=R d$ is essential. But since $R$ is left Goldie, the element $d$ is regular, so $R d$ is projective, being isomorphic to $R$. Thus $R x$ is projective since it is a summand of $R d$.

The following is the main result of this section.

THEOREM 5. Let $R$ be a semi-prime principal left ideal ring which is right Goldie. Let $Q$ be its classical quotient ring. Then the following are equivalent:

1. $(Q / R) \oplus R$ contains a copy of every simple right $R$-module.

2. $R$ is a principal right ideal ring.

3. Each maximal right ideal of $R$ is principal.

4. $(Q / R) \oplus Q$ is an injective cogenerator as a right $R$-module.

5. Countably generated right ideals are projective.

6. $R / A$ is an artinian module for every essential left ideal $A$.

Proof. 1 implies 2: By Lemma 4, $R$ is left semi-hereditary, thus Theorem 2 applies and we conclude that $R$ is right noetherian. But 3.7 of [7] asserts that a semi-prime two-sided noetherian ring is principal on the left if and only if it is principal on the right. Thus we have 2.

2 implies 3: Trivial.

3 implies 1: Let $M=c R$. If $c R$ is large then $c$ is regular and we have an embedding $0 \rightarrow R / c R \rightarrow Q / R$ by $1+c R \mapsto c^{-1}+R$. If $c R$ 
is not large, then $R=c R \oplus S$ and we have an embedding $0 \rightarrow R / c R \rightarrow R$.

3 implies 4: By 3 implies 2, $R$ is principal on the right, and by Lemma $4, R$ is right hereditary. Thus, since $Q_{R}$ is always injective, $Q / R$ and hence $(Q / R) \oplus Q$ is injective. Then 3 implies 1 makes $(Q / R) \oplus Q$ a cogenerator.

4 implies 1: $Q$ is an essential extention of $R$ as a right $R$ module, thus any simple right $R$-module which is contained in $Q$ is also contained in $R$.

2 implies 5: Lemma 4.

5 implies 2: It is well known that the hypotheses on $R$ imply that the right singular ideal of $R$ is 0 . Thus, by Corollary 1 of [8], projective right ideals are finitely generated. So, countably generated right ideals are finitely generated, and $R$ is noetherian. The conclusion now follows from 3.7 of [7].

2 implies 6: $A$ will contain a regular element $x$, and there is an exact sequence $R / R x \rightarrow R / A \rightarrow 0$. Now $R / R x$ is artinian by Lemma 3, since $R / x R$ is noetherian, so $R / A$ is artinian.

6 implies 2: By 3.6 of [7], finitely generated right ideals are principal, thus any ascending chain of finitely generated right ideals may be written as a chain of principals. Let $x_{1} R \subset x_{2} R \cdots$ be such a chain. Let $T=\cup x_{i} R$ and $K$ be a right ideal maximal with respect to the property that $T \cap K=0$. Then $T \oplus K$ is essential, so by the Goldie theorems there is a regular element $x_{n} r+k$ in $T \oplus K$. We then have an ascending chain $x_{n} R \oplus k R \subset x_{n+1} R \oplus k R \ldots$ which must stop by Lemma 3 and the hypothesis.

CoRollary 6. Let $R$ be a semi-prime principal left ideal ring which is right Goldie. If $R$ has a unique simple left $R$-module, then $R$ is right principal.

Proof. Let $R c$ generate a maximal left ideal. If $R c$ is large, $c$ is regular, and by the anti-isomorphism of Lemma 3, $c R$ generates a maximal right ideal, so $R / c R$ may be embedded in $Q / R$.

If $R c$ is not large then $R$ contains a minimal left ideal $S$. Then $I=S R$ must be all of $R$. To see this, suppose not and let $I \subset M$, where $M$ is a maximal left ideal. Then, $R / M \not S$ since $I S=S(R$ is semi-prime) but $I \cdot R / M=0$. This shows that $R$ is simple artinian since it will be a homomorphic image of finitely many copies of ${ }_{R} S$ (write $1=\Sigma s_{i} r_{i}$ ) and has a unique simple left $R$-module. Thus $R$ will be right principal.

Theorem 2 and its consequence, Theorem 5, have an interesting specialization.

Proposition 7. Let $R$ be a semi-prime principal left ideal ring 
which is right Goldie. Let $Q$ be the classical quotient ring of $R$, and $A$ be an essential right ideal, then the following are equivalent.

1. There is an embedding $0 \rightarrow R / A \rightarrow Q / R$.

2. A is principal.

Proof. 2 implies 1 is easy, since by the Goldie theorems $A$ will have a generator which is regular.

1 implies 2: Let $d \in Q$ be such that $A=\{r \in R \mid d r \in R\}$. Let $A^{-1}=$ $\{q \in Q \mid q A \subset R\}$. Then, $A$ contains a regular element $x$, and $A^{-1} x$ is a left ideal generated by $q x$. Now, since $1 \in A^{-1}, x \in A^{-1} x$, so there is an $s \in R$ with $s q x=x$. Thus, $s$ is regular and $q=s^{-1}$ since we may cancel $x$. Thus, $s^{-1} A \subset R$, or $A \subseteq s R$,

On the other hand, we have that $d x=p s^{-1} x$, which implies that $d s s \in R$, when we cancel $x$. Thus, $s \in A$, and the result follows.

REMARK. If $R$ is a principal left ideal domain which is right Ore with clasical quotient division ring $D$, then the previous results give here equivalence of the following (provided $D \neq R$ ):

1. $D / R$ contains a copy of every simple right $R$-module.

2. $D / R$ contains a copy of every cyclic right $R$-module.

3. $R$ is right principal.

4. $R / A$ is an artinian module for every nonzero left ideal $A$.

We close with a proposition which shows that one can say something about the right side of a principal left ideal domain with the Ore condition. Recall, a domain is a 2-fir if, whenever $R a \cap R b \neq 0$, $R a \cap R b$ and $R a+R b$ are principal. This definition is left-right symmetric [6].

Proposition 8. Let $R$ be a 2-fir with the ascending chain condition on principal left ideals, then the intersection of any number of principal right ideals is again principal.

Proof. By Lemma 3.3 of [6], any finite intersection of principals is principal. Consider the descending chain $b_{1} R \supset b_{1} R \cap b_{2} R \ldots$ of principal right ideals. If this intersects to zero we are done. If not, then the intersection contains $x \neq 0$, and by Lemma 3 the chain stops.

In particular, in a principal left ideal domain, the intersection of any number of principal right ideals is again principal.

We wish to thank the referee for many suggestions, which lead to often substantial improvements of our original results, and which we have incorporated in the revised manuscript. 


\section{REFERENCES}

1. P. M. Cohn, Free rings and their relations, Academic Press, 1971.

2. - Noncommutative unique factorization domains, Trans. Amer. Math. Soc., 109 (1963), 313-331.

3. — Free associative algebras, Bull. London Math. Soc., 1 (1969), 1-39.

4. A. V. Jategaonkar, Left Principal Ideal Rings, Springer Lecture Notes in Mathematics 123, Springer-Verlag, Berlin.

5. - A counterexample in ring theory and homological algebra, J. Algebra, 12 (1969), 418-440.

6. Irving Kaplansky, Rings and Fields, University of Chicago Press (1969).

7. J. C. Robson, Rings in which finitely generated right ideals are principal, Proc. London Math. Soc., Ser. 3, 17 (1966), 617-628.

8. F. Sandomierski, Nonsingular rings, Proc. Amer. Math. Soc., 19 (1968), 225-230.

9. D. G. Webber, Ideals and modules of simple noetherian hereditary rings, J. Algebra, 16 (1970), 239-242.

Received November 15, 1971 and revised form May 5, 1972.

UNIVERSITY OF IOWA

AND

Columbia UNIVERSITY 



\section{PACIFIC JOURNAL OF MATHEMATICS}

EDITORS

\author{
H. SAMELSON \\ Stanford University \\ Stanford, California 94305 \\ C. R. HOBBY \\ University of Washington \\ Seattle, Washington 98105
}

\author{
J. DUGUNDJI \\ Department of Mathematics \\ University of Southern California \\ Los Angeles, California 90007 \\ RICHARD ARENS \\ University of California \\ Los Angeles, California 90024
}

\section{ASSOCIATE EDITORS}

E. F. BECKENBACH

B. H. NeUManN

F. WOLF

K. YosHIDA

\section{SUPPORTING INSTITUTIONS}

UNIVERSITY OF BRITISH COLUMBIA

CALIFORNIA INSTITUTE OF TECHNOLOGY

UNIVERSITY OF CALIFORNIA

MONTANA STATE UNIVERSITY

UNIVERSITY OF NEVADA

NEW MEXICO STATE UNIVERSITY

OREGON STATE UNIVERSITY

UNIVERSITY OF OREGON

OSAKA UNIVERSITY
UNIVERSITY OF SOUTHERN CALIFORNIA

STANFORD UNIVERSITY

UNIVERSITY OF TOKYO

UNIVERSITY OF UTAH

WASHINGTON STATE UNIVERSITY UNIVERSITY OF WASHINGTON

AMERICAN MATHEMATICAL SOCIETY NAVAL WEAPONS CENTER 


\section{Pacific Journal of Mathematics}

\section{Vol. 45, No. $1 \quad$ September, 1973}

William George Bade, Complementation problems for the Baire classes .......... 1

Ian Douglas Brown, Representation of finitely generated nilpotent groups ........ 13

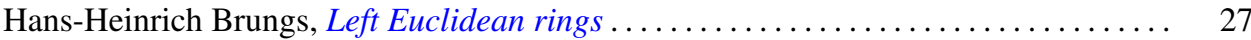

Victor P. Camillo and John Cozzens, A theorem on Noetherian hereditary rings ..... 35

James Cecil Cantrell, Codimension one embeddings of manifolds with locally flat

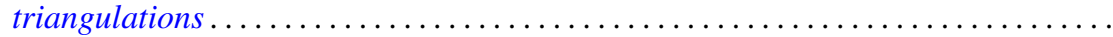

L. Carlitz, Enumeration of up-down permutations by number of rises . . . . . . . . . .

Thomas Ashland Chapman, Surgery and handle straightening in Hilbert cube

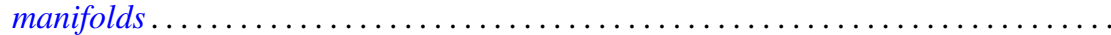

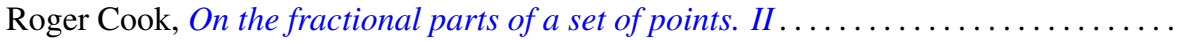

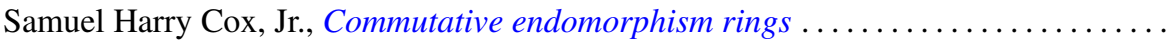

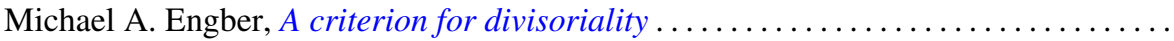

Carl Clifton Faith, When are proper cyclics injective . . . . . . . . . . . . . . 97

David Finkel, Local control and factorization of the focal subgroup . . . . . . . . . 113

Theodore William Gamelin and John Brady Garnett, Bounded approximation by

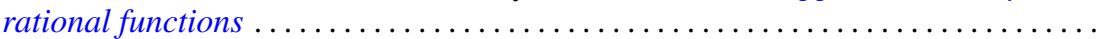

Kazimierz Goebel, On the minimal displacement of points under Lipschitzian

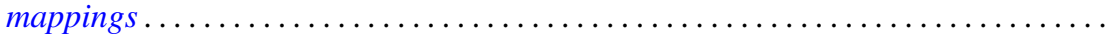

Frederick Paul Greenleaf and Martin Allen Moskowitz, Cyclic vectors for representations associated with positive definite measures: nonseparable

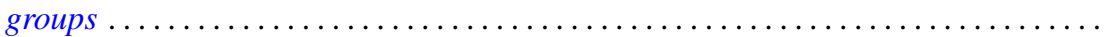

Thomas Guy Hallam and Nelson Onuchic, Asymptotic relations between perturbed linear systems of ordinary differential equations .

David Kent Harrison and Hoyt D. Warner, Infinite primes of fields and completions. .

James Michael Hornell, Divisorial complete intersections . ......

Jan W. Jaworowski, Equivariant extensions of maps ..............

John Jobe, Dendrites, dimension, and the inverse arc function .. .

Gerald William Johnson and David Lee Skoug, Feynman integrals of non-factorable

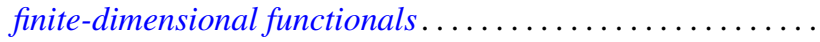

Dong S. Kim, A boundary for the algebras of bounded holomorphic functions ...... 269

Abel Klein, Renormalized products of the generalized free field and its derivatives ... 275

Joseph Michael Lambert, Simultaneous approximation and interpolation in $L_{1}$ and

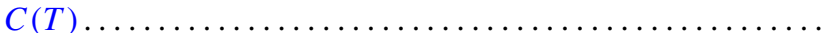

Kelly Denis McKennon, Multipliers of type $(p, p)$ and multipliers of the group

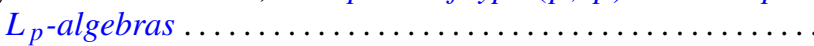

William Charles Nemitz and Thomas Paul Whaley, Varieties of implicative

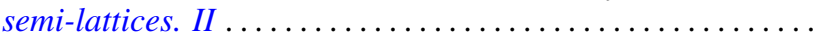

Donald Steven Passman, Some isolated subsets of infinite solvable

Norma Mary Piacun and Li Pi Su, Wallman compactifications on E-completely

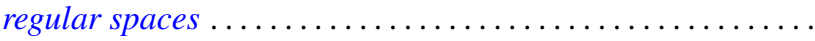

Jack Ray Porter and Charles I. Votaw, $S(\alpha)$ spaces and regular Hausdorff extensions....

Gary Sampson, Two-sided $L_{p}$ estimates of convolution transforms .

Ralph Edwin Showalter, Equations with operators forming a rig
Raymond Earl Smithson, Fixed points in partially ordered sets .

Victor Snaith and John James Ucci, Three remarks on symmetric products and

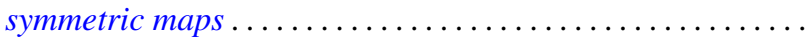

